удк 517.97

Н. В. Антипина

Байкальский государственный университет, 2. Иркутск, Российская Федеращия

\title{
ВЛИЯНИЕ ИНВЕСТИЦИОННОЙ СОСТАВЛЯЮЩЕЙ НА ЭКОНОМИЧЕСКИЕ ПОКАЗАТЕЛИ МАЛЫХ И СРЕДНИХ ФИРМ
}

\begin{abstract}
АНнОтАЦИЯ. В современных экономических условиях малые и средние предприятия подвержены воздействию многих негативных факторов риска. Малые предприятия уязвимы в силу отсутствия фундаментального финансового планирования и низкой материальной прочности. Очевидна необходимость доступности финансовых средств для развития бизнеса и открытия новых направлений, в том числе государственная поддержка через финансирование и предоставление государственных заказов. Поскольку собственники малого и среднего бизнеса не имеют влияния на вышеперечисленные факторы, то им лучше сосредоточиться на поиске новых подходов к управлению предприятиями. Целесообразно использование экономико-математических методов с акцентом на оптимизацию стратегии управления фирмой. В настоящее время это направление разработано недостаточно. В данной статье предпринята попытка частично восполнить этот пробел посредством качественного анализа экономико-математической модели динамики деятельности малых фирм.

кЛючЕВЫЕ СЛОВА. Малое предпринимательство; финансирование; инвестиции; динамика; экономико-математическое моделирование; оптимальное управление. ИНФОРМАЦИЯ О СТАТЬЕ. Дата поступления 28 апреля 2017 г.; дата принятия к печати 2 мая 2017 г.; дата онлайн-размещения 21 июня 2017 г.
\end{abstract}

N. V. Antipina

Baikal State University, Irkutsk, Russian Federation

\section{INFLUENCE OF INVESTMENT CONSTITUENT ON ECONOMIC INDICATORS OF SMALL AND MEDIUM-SIZED FIRMS}

\begin{abstract}
In the current economic environment small and medium-sized businesses are subject to impacts of numerous negative risk factors. Small businesses are vulnerable due to absence of fundamental financial planning and low material stability. There is an evident need for availability of financial funds for developing business and opening new directions, including governmental support through financing and offering public contracts. As long as owners of small and medium-sized businesses have no influence on the above-said factors, it is better for them to concentrate on search for new approaches to business management. It makes sense to use economic and mathematical methods with a focus on optimization of company management strategy. This article makes an attempt to partly fill up this gap by means of qualitative analysis of the economical and mathematical model for dynamics of small business activities.

KEYWORDS. Small business; financing; investments; dynamics; economic-mathematical modeling; optimal control.

ARTICLE INFO. Received April 28, 2017; accepted May 2, 2017; available online June 21, 2017.
\end{abstract}

Финансовые источники развития малого и среднего бизнеса. В экономике любой страны и в мировой экономике в целом значимым звеном являются малые предприятия. Благодаря их деятельности невостребованные крупными предприятиями производственный, научно-технический и трудовой потенциалы используются более эффективно.

(C) Н. В. Антипина, 2017

\section{Baikal Research Journal}

электронный научный журнал Байкальского государственного университета 
Малый бизнес представляет собой предпринимательскую деятельность, которую осуществляют субъекты свободных экономических отношений с соблюдением требований, установленных законами, органами государственного управления и надзорными органами. Основными критериями отнесения того или иного предприятия или предпринимателя к категории малого бизнеса являются среднегодовая численность работников, выручка за год и размер прибыли. Хотя по данным Всемирного банка, критериев, по которым предприятия, организации и предприниматели получают статус малого бизнеса, в мире по совокупности насчитывается около 50 [1].

Роль и место малого предпринимательства в национальной экономике ярче всего проявляется в присущих ему функциях. Рассматривая функции малого предпринимательства в рыночной экономике, надо отметить, в частности, их следующие социально-экономические функции [1-4]:

- формирование конкурентной среды;

- демонополизация, приватизация и разгосударствление экономики;

- заполнение недоступных и малоэффективных для крупных производственных и торговых фирм рыночных ниш;

- перестройка промышленной структуры;

- ограничение конкуренции со стороны иностранных производителей;

- новаторская экспортная деятельность;

- обеспечение занятости населения и подготовка кадров;

- инновационная деятельность.

Двойственность экономической природы малых предприятий проявляется в их гибкости и мобильности с одной стороны, и в слабости, маломощности и небольшом запасе прочности - с другой. Это позволяет говорить о более сложных и менее стабильных условиях функционирования малого бизнеса по сравнению с крупными экономическими предприятиями и фирмами.

К сожалению, последнее десятилетие наблюдается спад роста числа малых предприятий. По сравнению с мировой практикой, как известно, «насыщенность» экономики России малыми предприятиями оставляет желать лучшего:

- число малых предприятий на душу населения в России на порядки отстает от развитых западных стран;

- малый бизнес в российской экономике лишен возможности выполнять основные роли и функции, отведенные ему по статусу [1].

Причины обозначенных проблем кроются в малом размере стартового капитала и недостатке источников поддержки и развития малого бизнеса $[3 ; 5 ; 6]$.

Главной причиной сложившейся ситуации является отсутствие достаточных инвестиционных вливаний. Основными источниками финансирования на западе являются банковские кредиты и государственная финансовая поддержка. В российских экономических условиях финансовая поддержка государства практически отсутствует, а кредиты не балуют доступностью (из-за завышенной процентной ставки, большого риска малого предпринимательства, проблем ссудного залога), что отражено в табл. 1.

Неблагоприятный инвестиционный климат для малых предприятий обусловлена следующими факторами:

1. Воздействие внешней среды (инфляция, конкуренция на рынке, высокие барьеры внедрения на рынок, налоги, недоверие кредитно-инвестиционных организаций).

2. Внешние инвестиционные ресурсы (высокие инвестиционные риски, малая величина и доступность некоторых видов ресурсов, высокие ставки процентов по кредиту).

\section{Baikal Research Journal}


3. Внутренние инвестиционные ресурсы (недостаточная величина стартового капитала и собственных средств развития).

Объем кредитов, предоставленных субъектам

Таблица 1 малого и среднего предпринилательства, млн р.

\begin{tabular}{|c|r|r|}
\hline \multirow{2}{*}{ Год } & \multicolumn{2}{|c|}{ Объем предоставленных кредитов, всего } \\
\cline { 2 - 3 } & $\begin{array}{c}\text { субъектам малого и среднего } \\
\text { предпринимательства }\end{array}$ & $\begin{array}{c}\text { из них, индивидуальным } \\
\text { предпринимателям }\end{array}$ \\
\hline 2009 & 3002887 & 209900 \\
\hline 2010 & 4704715 & 412962 \\
\hline 2011 & 6055744 & 556055 \\
\hline 2012 & 6942525 & 653572 \\
\hline 2013 & 8064759 & 691028 \\
\hline 2014 & 7640594 & 582582 \\
\hline 2015 & 5460273 & 308247 \\
\hline 2016 & 5302130 & 342727 \\
\hline 2017 (январь) & 304333 & 20931 \\
\hline
\end{tabular}

Составлено по данным: Банк России. Статистика. URL: http://www.cbr.ru/statistics/udstat. aspx ?TblID $=302-17$.

Стартовый капитал - величина первоначальных инвестиционных вложений, без которой малый бизнес не только не выгоден, но и нежизнеспособен - в значительной степени определяется низким уровнем благосостояния среднего класса населения, решившего начать собственное дело, и поэтому не может обеспечивать достаточный уровень функционирования малого предприятия [3; $5 ; 6]$.

Внутренние источники развития (страховые, резервные и амортизационные фонды, прибыль) также нельзя рассматривать как серьезную финансовую основу, позволяющую малому бизнесу развиваться и выживать в сложных условиях становления рыночных отношений.

По этим причинам использование банковских кредитов влечет за собой задолженности малых предприятий, которые с течением лет растут (табл. 2).

Задолженность, по кредитал, предоставленныл субъектал

Таблица 2 малого и среднего предпринилательства, млн р.

\begin{tabular}{|c|r|r|r|r|}
\hline \multirow{2}{*}{ Год } & \multicolumn{2}{|c|}{ Задолженность всего } & \multicolumn{2}{c|}{ В том числе просроченная } \\
\cline { 2 - 5 } & $\begin{array}{c}\text { субъектам малого и } \\
\text { среднего предприни- } \\
\text { мательства }\end{array}$ & $\begin{array}{c}\text { из них, индиви- } \\
\text { дуальным пред- } \\
\text { принимателям }\end{array}$ & $\begin{array}{c}\text { субъектам малого и } \\
\text { среднего предприни- } \\
\text { мательства }\end{array}$ & $\begin{array}{c}\text { из них, индиви- } \\
\text { дуальным пред- } \\
\text { принимателям }\end{array}$ \\
\hline 2009 & 200111 & 20987 & 187751 & 20725 \\
\hline 2010 & 284048 & 27313 & 271046 & 26856 \\
\hline 2011 & 314753 & 27121 & 304485 & 26609 \\
\hline 2012 & 377247 & 24618 & 365059 & 24269 \\
\hline 2013 & 365445 & 32900 & 356115 & 32610 \\
\hline 2014 & 394388 & 52061 & 378751 & 51785 \\
\hline 2015 & 666199 & 70070 & 632928 & 69443 \\
\hline 2016 & 635992 & 62202 & 619216 & 61936 \\
\hline 2017 январь) & 637160 & 52632 & 621035 & 61936 \\
\hline
\end{tabular}

Составлено по данным: URL: http://www.cbr.ru/statistics/udstat.aspx?TblID=302-17.

Основными финансовыми источниками для предпринимателей РФ являются внутренние источники:

- частный кредит $(15 \%)$;

- личные средства учредителей (20\%);

\section{Baikal Research Journal}

электронный научный журнал Байкальского государственного университета 
- прибыли от собственной деятельности (60\%).

Причины их использования очевидны: доступность и надежность. Доля внешних ресурсов (кредиты банков, бюджетные ссуды и др.) составляет не более $5 \%$.

Внешними источниками заимствований пользуется менее половины всех малых предприятий, при этом они неизбежно сталкиваются с проблемами, связанными со слабым развитием рынка финансовых услуг [7].

Финансовая поддержка государства, к сожалению, малозначима для сектора малых предприятий, поскольку единовременна, невелика по размеру и, в большинстве случаев, не доходит до адресата $[6 ; 8]$.

Так, в 2014 г. в поддержку МСП было выделено около 20 млрд р., а уже в 2015 - 17 млрд р. В 2016 г. из федерального бюджета планировалось предоставить финансовую помощь в размере почти 15 млрд р., но по факту получилось 11 млрд р.

В 2017 г. эта тенденция к уменьшению финансовой помощи сохраняется: государство готово предоставить всего 7,5 млрд р.

Поэтому тем, кто рассчитывает на поддержку своего бизнеса, придется приложить немало усилий, чтобы ее получить.

Структура расходов на помощь малому бизнесу в 2017 г. отражена в табл. 3.

Структура расходов на помощь малому бизнесу в 2017 г. , млрд р.

\begin{tabular}{|c|c|}
\hline Расходы & Сумма \\
\hline Образование инфраструктуры для развития малого и среднего предпринимательства & 3,06 \\
\hline Мероприятия по поддержке монопрофильных муниципальных образований & 0,74 \\
\hline Создание и развитие информационно-консультационной поддержки & 0,72 \\
\hline $\begin{array}{l}\text { Создание инфраструктуры поддержки малого и среднего предпринимательства, осу- } \\
\text { ществляющей свою деятельность в области инноваций и промышленного производства }\end{array}$ & 0,69 \\
\hline Завершение объектов капитального строительства & 1,6 \\
\hline Содействие развитию молодежного предпринимательства & 0,23 \\
\hline Создание многофункциональных центров для бизнеса & 0,135 \\
\hline
\end{tabular}

В силах государства:

- запустить механизмы финансовой поддержки;

- применить весь спектр технологий привлечения средств в сферу малого предпринимательства;

- сделать систему предоставления гарантий и бюджетных ресурсов более понятной и доступной предпринимателям;

- обучить предпринимателей современным методам заимствования;

- переориентировать систему финансирования на активное использование внебюджетных источников [8].

Сущность экономико-математического моделирования. Отметим, что решение проблем кредитно-инвестиционной политики в секторе малого бизнеса значительно осложняется и теоретико-методическими трудностями. Как правило, при попытке решить эти проблемы ограничиваются качественными методами анализа, не учитывая, что данный вопрос требует комплексного подхода, и, как следствие, - применения количественных методов. Речь идет о моделировании и применении экономико-математических методов, учитывающих специфику исследуемого экономического объекта - малого предприятия.

Перечислим некоторые прикладные задачи экономико-математического моделирования [9]:

\section{Baikal Research Journal}


- анализ экономических процессов, объектов и ситуаций;

- прогнозирование сценариев экономических процессов;

- разработка управленческих решений на любом экономическом уровне.

$\kappa$ наиболее распространенным экономико-математическим моделям относятся модели оптимизации, которые в основном применяются в микроэкономике (минимизация издержек, максимизация полезности потребителя, прибыли фирмы). В макроэкономике результатом выбора оптимальной стратегии экономических субъектов оказывается некоторое состояние равновесия.

К основным признакам оптимизационных моделей относят следующие:

- наличие одного или нескольких критериев оптимальности (например, в экономических задачах типичными являются следующие: минимум издержек, максимум дохода (прибыли), минимум времени транспортировки продукции и т. д.);

- система ограничений, которая следует из содержательного смысла задачи, и представляет собой набор соотношений (уравнений, в том числе дифференциальных, и/или неравенств) [10].

Под построением экономико-математической модели понимается математическая формализация исследуемой экономической ситуации, с учетом ее специфики и большинства влияющих на нее внешних и внутренних факторов. Экономические решения относятся к той категории решений, следствием которых являются прибыли или убытки. Поэтому до принятия решения приходится анализировать ситуацию, определять критерии выбора и искать наилучшие (оптимальные) решения.

Заметим, что данные, полученные в результате экономико-математического моделирования, не следует воспринимать как «готовый рецепт» управленческого решения. Они, в большей степени, должны рассматриваться экономическими субъектами как рекомендации. Окончательное принятие управленческого решения осуществляется человеком.

Итак, экономико-математическое моделирование является значимым компонентом в процессе прогнозирования и планирования экономической деятельности, а также принятия управленческих решений, но все же - одним из многих.

В настоящее время экономико-математические методы в направлении малого бизнеса разработаны недостаточно. Данная статья, в некоторой степени, восполняет этот пробел, предлагая анализ экономико-математической модели функционирования малого предприятия.

Модель динамики малого предприятия, использующего самофинансирование и внешние инвестиции

Постановка задачи. Рассмотрим малое предприятие, которое функционирует в течение периода времени $[0 ; T]$ за счет собственных источников прибыли $M(t)$, а также за счет использования внешних инвестиций $I(t)$, поступающих либо из фондов поддержки малого бизнеса, либо являющихся формой финансовой поддержки в рамках государственной программы [6]. Будем считать, что основные производственные фонды $A(t)$ предприятия (в стоимостном выражении) являются единственным определяющим выпуск продукции фактором, и начальный уровень этих фондов в момент времени $t=0$ равен $A_{0}$. Поскольку технология выпуска продукции, как правило, меняется через 30-40 лет, то можно предположить, что малое предприятие осуществляет выпуск продукции при неизменной технологии, что гарантирует его постоянную фондоотдачу $f$.

Деятельность малого предприятия описывает однофакторная производственная функция $P(t)$ типа Леонтьева [11], связанная линейной зависимостью с основными производственными фондами:

$$
P(t)=f A(t)
$$

\section{Baikal Research Journal}

электронный научный журнал Байкальского государственного университета 
Общую прибыль предприятия $M^{\circ \sigma}(t)$ с учетом издержек производства определяет равенство

$$
M^{\circ б}(t)=(1-c) P(t),
$$

где $c$ - это удельная себестоимость продукции.

Кроме того, малое предприятие выплачивает налог на объем выпуска продукции и налог на прибыль по ставкам $\tau_{1}$ и $\tau_{2}$ соответственно. Таким образом, упрощенный механизм налогообложения учитывается в виде соотношения

$$
N(t)=\tau_{1} P(\mathrm{t})+\tau_{2} k(1-\xi) M(t),
$$

где $\xi=\xi(t)-$ доля чистой прибыли, отчисляемой на реинвестирование в каждый момент времени $t \in[0 ; T]$ (очевидно, $0 \leq \xi(t) \leq 1$ ); $k-$ постоянная доля реинвестируемых средств чистой прибыли, не имеющих льгот по налогообложению, $0<k \leq 1$ (может оцениваться статистическими методами); $M(t)$ - объем чистой прибыли за вычетом налоговых отчислений (3).

Легко видеть, что $M(t)$ выражается следующим соотношением:

$$
M(t)=M^{\circ \sigma}(t)-N(t) .
$$

Эффективность работы малого предприятия определяется динамикой основных производственных фондов $A(t)$ посредством самофинансирования и внешних инвестиционных вложений $I(t)$ и формализуется в виде дифференциального уравнения

$$
\frac{d A(t)}{d t}=\xi M(t)+I(t), \quad A(0)=A_{0} .
$$

Итак, модель динамики функционирования малого предприятия имеет вид соотношений (1)-(5).

Произведем ряд преобразований над ними в целях упрощения.

Подставляя выражение (2) для общей прибыли $M^{\circ \sigma}(t)$ и суммы налоговых отчислений $N(t)$ вида (3) в соотношение (4), получим следующее:

$$
M(t)=\left(1-c-\tau_{1}\right) P(t)-\tau_{2} k(1-\xi) M(t) .
$$

Отсюда, учитывая выражение (1) для производственной функции $P(t)$, явно найдем функцию чистой прибыли

$$
M(t)=\frac{1-c-\tau_{1}}{1+\tau_{2} k(1-\xi)} f A(t)
$$

и подставим ее в уравнение динамики (5).

В результате уравнение (5) примет вид

$$
\frac{d A(t)}{d t}=\alpha A(t)+I(t), \quad A(0)=A_{0} .
$$

где коэффициент

$$
\alpha=\alpha(t)=\frac{\left(1-c-\tau_{1}\right) \xi f}{1+\tau_{2} k(1-\xi)}>0
$$

не является постоянным (зависит от времени $t$ ), поскольку $\xi=\xi(t)$, и учитывает влияние режима налогообложения на реинвестирование.

Таким образом, в дифференциальном уравнении (7) учтены все соотношения модели (1)-(5).

Форлализация задачи оптимального управления. Описанная модель функционирования малого предприятия [6] служит благодатной почвой для формализации задачи оптимального управления $[12 ; 13]$, если считать, что малое предпри-

\section{Baikal Research Journal}


ятие имеет целью максимизацию дисконтированной чистой прибыли за период времени $[0 ; T]$ за счет управления долей реинвестирования.

Пусть выполняются все предположения этой модели.

Основой задачи оптимального управления служит динамическое уравнение (7), которое с математической точки зрения является линейным неоднородным дифференциальным уравнением первого порядка.

Для лаконичности записи будущей задачи оптимального управления введем новые обозначения

$$
\beta=\tau_{2} k, \gamma=\left(1-c-\tau_{1}\right) f .
$$

Заметим, что, в соответствии с экономическим смыслом параметров базовой модели, оба коэффициента постоянны и $\beta>0, \gamma>0$

Тогда выражение (6) для чистой прибыли $M(t)$ запишется в виде

$$
M(t)=\frac{\gamma}{1+\beta(1-\xi)} A(t) .
$$

Считая целью малого предприятия максимизацию дисконтированной чистой прибыли, сформируем критерий качества

$$
J(\xi)=\int_{0}^{T} M(t) e^{-r t} d t \rightarrow \max ,
$$

где $r$ - коэффициент дисконтирования.

Таким образом, приходим к задаче оптимального управления вида

$$
\begin{gathered}
J(\xi)=\int_{0}^{T} M(t) e^{-r t} d t=\int_{0}^{T} \frac{\gamma A(t)}{1+\beta(1-\xi(t))} e^{-r t} d t \rightarrow \max , \\
\frac{d A(t)}{d t}=\frac{\gamma \xi}{1+\beta(1-\xi)} A(t)+I(t), \quad A(0)=A_{0}, \\
0 \leq \xi(t) \leq 1, t \in[0 ; T], \\
\beta>0, \gamma>0 .
\end{gathered}
$$

В этой задаче управляющей переменной является $\xi(t)$, причем множество ее допустимых значений ограничено и определяется неравенством (10), а переменной состояния $-M(t)$, удовлетворяющая динамическому уравнению (9) с начальным условием.

Задача (8)-(10) является простейшей задачей оптимального управления с интегральным критерием качества (8) на фиксированном отрезке времени $[0 ; T]$.

Исследование модели. Проведем исследование задачи (8)-(10) с помощью принципа максимума Понтрягина [12-14].

Запишем функцию Понтрягина

$$
H(t, A, \psi, \xi)=\psi\left(\frac{\gamma \xi}{1+\beta(1-\xi)} A(t)+I(t)\right)+\frac{\gamma A(t)}{1+\beta(1-\xi)} e^{-r t}
$$

и на ее основе сформируем сопряженное уравнение

$$
\dot{\psi}=-H_{A}=-\frac{\gamma \xi}{1+\beta(1-\xi)} \psi-\frac{\gamma}{1+\beta(1-\xi)} e^{-r t}, \quad \psi(T)=0 .
$$

Функция переключения управления представляет собой частную производную функции Понтрягина по управлению $\xi$ и имеет следующий вид:

\section{Baikal Research Journal}




$$
h(t, A, \psi, \xi)=H_{\xi}=\frac{\gamma(1+\beta) A}{(1+\beta(1-\xi))^{2}} \psi+\frac{\beta \gamma A}{(1+\beta(1-\xi))^{2}} e^{-r t} .
$$

Для того, чтобы избавиться от множителя $e^{-r t}$, сделаем замену сопряженной переменной $\lambda(t)=\psi(t) e^{r t}$ (или, что то же самое, $\psi(t)=\lambda(t) e^{-r t}$ ).

В результате новая сопряженная переменная удовлетворяет дифференциальному уравнению

$$
\dot{\lambda}=\left(r-\frac{\gamma \xi}{1+\beta(1-\xi)}\right) \lambda-\frac{\gamma}{1+\beta(1-\xi)}, \lambda(T)=0 .
$$

Функция переключения (11), с учетом сделанной замены, примет вид

$$
h(t, A, \lambda, \xi)=\frac{\gamma A(1+\beta)}{(1+\beta(1-\xi))^{2}} \lambda+\frac{\beta \gamma A}{(1+\beta(1-\xi))^{2}}=\frac{\gamma A[(1+\beta) \lambda+\beta]}{(1+\beta(1-\xi))^{2}} .
$$

Очевидно, функция (13) не линейна по управлению $\xi$. Выясним, достигает ли она своего максимального значения на отрезке [0; 1]. Для этого потребуются элементы исследования этой функции на данном отрезке.

Легко видеть, что $h$ определена при всех

Поскольку значение

$$
\xi \neq \frac{1+\beta}{\beta}
$$

$$
\frac{1+\beta}{\beta}>1,
$$

что выходит за пределы отрезка $[0 ; 1]$, то на всем отрезке функция переключения определена. Далее заметим, что $h \neq 0, \forall \xi \in[0 ; 1]$, а знак $h$ определяется знаком скобки $[(1+\beta) \lambda+\beta]$, поскольку $\gamma>0$ и $\mathbf{A}>0$ по экономическому смыслу. Значит, возможны два случая поведения функции переключения $h$, изображенные на рисунке.

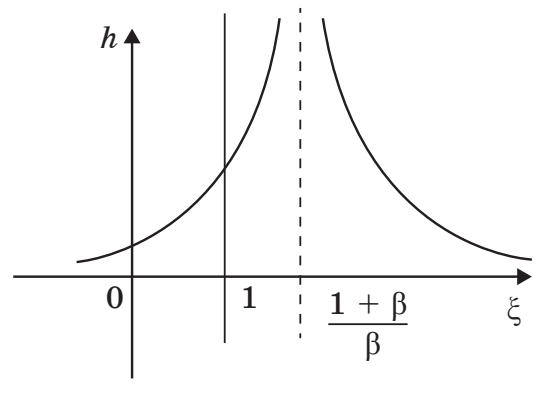

$a$

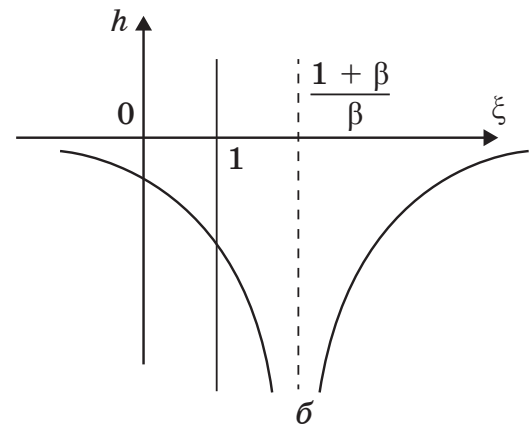

б

Поведение функиии переключения $h: a-n p u ~ h>0 ; \sigma-n p u ~ h<0$

Напомним, что $\xi(t)$, удовлетворяющее принципу максимума таково:

$$
\xi(t)=\left\{\begin{array}{cc}
0, & \text { если } h<0, \\
1, & \text { если } h>0, \\
\forall \xi \in[0 ; 1], & \text { если } h=0 .
\end{array}\right.
$$

\section{Baikal Research Journal}


Однако, проведенное исследование функции переключения позволяет утверждать, что функция переключения "держит знак» на всем временном промежутке $[0 ; T]$, что означает отсутствие точек переключения и особого интервала управления $\xi$, соответствующих $h=0$. Поэтому управление $\xi(t)=0 \vee 1=c o n s t$ на отрезке $[0 ; 1]$.

Для определения постоянного экстремального значения управления исследуем поведение сопряженной функции и функции переключения в левой окрестности $O(T)=(\theta ; T)$ точки Т.

Учитывая, что $\lambda(T)=0$, можем сделать вывод об убывании $\lambda(t)$ в окрестности $O(T)$, так как

$$
\left.\dot{\lambda}\right|_{O(T)} \approx-\frac{\gamma}{1+\beta(1-\xi)}<0 .
$$

Следовательно, $\lambda(t)>0$ в окрестности $O(T)$, а значит и

$$
\left.h\right|_{o(T)} \approx \frac{\beta \gamma A}{(1+\beta(1-\xi))^{2}}>0,
$$

и $\xi(t)=1$ в этой окрестности.

Подставляя $\xi=1$ в (12), получим следующее уравнение для двойственной переменной:

$$
\dot{\lambda}=(r-\gamma) \lambda-\gamma, \lambda(T)=0 .
$$

Согласно теории обыкновенных дифференциальных уравнений [15], это линейное неоднородное дифференциальное уравнение первого порядка, имеет следующее решение:

$$
\lambda(t)=\frac{\gamma}{r-\gamma}\left(1-e^{(r-\gamma)(t-T)}\right), \quad \forall t \in O(T) .
$$

Легко проверить, что сопряженная переменная не только имеет смысл, но и положительна $(\lambda(t)>0)$ при выполнении условия

$$
r-\gamma \neq 0 \text {. }
$$

С учетом сохранения знака $h$ и соотношения (14), экстремальное управление таково: $\xi(t)=\xi * \equiv 1, \forall t \in[0 ; T]$.

Теперь несложно найти траекторию $A(t)$, решив дифференциальное уравнение (9) при $\xi \equiv 1$ :

$$
\frac{d A(t)}{d t}=\gamma A(t)+I(t), \quad A(0)=A_{0} .
$$

Это уравнение имеет следующее решение:

$$
A *(t)=e^{\gamma t}\left[A_{0}+\int_{0}^{t} I(z) e^{-\gamma z} d z\right], \quad \forall t \in[0 ; T] .
$$

Анализ рассмотренной экономико-математической моделей деятельности малых предприятий свидетельствует о том, что одних внутренних ресурсов недостаточно для обеспечения необходимых темпов роста предприятия. Замедленная динамика функционирования малого предприятия, которое использует лишь принцип самофинансирования, становится особенно отчетливой при постановке задачи, учитывающей процесс выбытия основных фондов.

Проведенное исследование модели и анализ источников финансирования малого бизнеса свидетельствует о том, что для достаточного уровня функционирования малого предприятия его инвестиционная стратегия должна быть консолидированной и основываться на аккумуляции средств из различных, как внутренних, так и внешних, источников.

\section{Baikal Research Journal}




\section{Список использованной литературы}

1. Акимов О. Ю. Малый и средний бизнес: эволюция понятий, рыночная среда, проблемы развития / О. Ю. Акимов. - М. : Финансы и статистика, 2004. - 192 с.

2. Бурлуткин Т. В. Анализ современного состояния и проблем функционирования малого предпринимательства в Российской Федерации [Электронный ресурс] / Т. В. Бурлуткин // Экономические исследования. - 2011. - № 6 (11). - Режим доступа: http:// ecsocman.hse.ru/text/36889790/.

3. Егорова Н. Е. Малый бизнес в России: экономический анализ и моделирование / Н. Е. Егорова, Е. Р. Майн. - М. : Центр. экон.-мат. ин-т РАН : Ин-т соц.-экон. проблем народонаселения РАН, 1997.

4. Горфинкель В. Я. Малый бизнес: организация, экономика, управление / В. Я. Горфинкель, В. В. Швандар. - М. : Юнити, 2007. - 432 с.

5. Пурыжова Л. В. Особенности финансирования малого и среднего бизнеса / Л. В. Пурыжова, С. Н. Клочко // Вопросы экономики и управления. - 2016. - № 2. - С. 64-67.

6. Егорова Н. Е. Дифференциальный анализ развития малых предприятий, использующих кредитно-инвестиционный ресурс / Н. Е. Егорова, С. Р. Хачатрян, М. А. Маренный // Аудит и финансовый анализ. - 2000. - № 4. - С. 11-37.

7. Литвак Е. Г. Влияние кризисных явлений в экономике Российской Федерации на малый бизнес / Е. Г. Литвак // Финансовая газета. - 2009. - № 6. - С. 25-27.

8. Талалаева И. Государственная поддержка малого и среднего бизнеса / И. Талалаева // Малая бухгалтерия. - 2010. - № 1. - С. 18.

9.Макарова С. И. Экономико-математические методы и модели / С. И. Макарова. М. : КноРус, 2009. -240 c.

10. Федосеев В. В. Экономико-математические методы и прикладные модели / В. В. Федосеев, А. Н. Гармаш, И. В. Орлова. - М. : Юрайт, 2012. - 328 с.

11. Клейнер Г. Б. Производственные функции: теория, методы, применение / Г. Б. Клейнер. - М. : Кн. по Требованию, 2012. - 240 с.

12. Кротов В. Ф. Методы и задачи оптимального управления / В. Ф. Кротов, В. И. Гурман. - М. : Наука, 1973. - 448 с.

13. Математическая теория оптимальных процессов / Л. С. Понтрягин, В. Г. Болтянский, Р. В. Гамкрелидзе, Е. Ф. Мищенко. - М. : Наука, 1983. - 393 с.

14. Дыхта В. А. Оптимальное управление в экономике: простейшие модели / В. А. Дыхта, Н. В. Антипина, О. Н. Самсонюк. - Иркутск : Иркут. гос. ун-т, 1998. - 115 с.

15. Егоров А. И. Обыкновенные дифференциальные уравнения с приложениями / А. И. Егоров. - 2-е изд., испр. - М. : Физматлит, 2005. - 384 с.

\section{References}

1. Akimov O. Yu. Malyi i srednii biznes: evolyutsiya ponyatii, rynochnaya sreda, problemy razvitiya Small and medium-sized businesses: evolution of concepts, market environment, problems of development]. Moscow, Finansy i statistika Publ., 2004. 192 p.

2. Burlutkin T. V. Analysis of current state of problems of small business functioning in the Russian Federation. Ekonomicheskie issledovaniya = Economic Research, 2011, no. 6 (11). Available at: http://ecsocman.hse.ru/text/36889790/. (In Russian).

3. Egorova N. E., Main E. R. Malyi biznes v Rossii: ekonomicheskii analiz i modelirovanie [Small business in Russia: economic analysis and modelling]. Moscow, Central Economics and Mathematics Institute of Russian Academy of Sciences Publ., Institute of Socio-Economic Studies of Population of the Russian Academy of Sciences Publ., 1997.

4. Gorfinkel V. Ya., Shvandar V. V. Malyi biznes: organizatsiya, ekonomika, upravlenie [Small business: arrangement, economy, management. Moscow, Yuniti Publ., 2007. 432 p.

5. Puryzhova L. V., Klochko S. N. Features of financing small and medium-sized businesses. Voprosy ekonomiki i upravleniya = Issues of Economics and Management, 2016, no. 2, pp. 64-67. (In Russian).

6. Egorova N. E., Khachatryan S. R., Marenny M. A. Differential analysis of development of small businesses that use credit-investment recourses. Audit $i$ finansovyi analiz $=$ Auditing and Financial Analysis, 2000, no. 4, pp. 11-37. (In Russian).

7. Litvak E. G. Impact of crisis phenomena on small business in the Russian Federation economy. Finansovaya gazeta = Financial Newspaper, 2009, no. 6, pp. 25-27. (In Russian).

\section{Baikal Research Journal}


8. Talalayeva I. Governmental support of small and medium-sized businesses Malaya bukhgalteriya $=$ Small Accounting, 2010, no. 1, p. 18. (In Russian).

9. Makarova S. I. Ekonomiko-matematicheskie metody i modeli [Economic and mathematical methods and models]. Moscow, KnoRus Publ., 2009. 240 p.

10. Fedoseyev V. V., Garmash A. N., Orlova I. V. Ekonomiko-matematicheskie metody $i$ prikladnye modeli [Economic and mathematical methods and application models]. Moscow, Yurait Publ., 2012. 328 p.

11. Kleiner G. B. Proizvodstvennye funktsii: teoriya, metody, primenenie [Industrial functions: theory, methods, use]. Moscow, Kniga po Trebovaniyu Publ., 2012. 240 p.

12. Krotov V. F., Gurman V. I. Metody $i$ zadachi optimal'nogo upravleniya [Methods and Optimal Control Tasks]. Moscow, Nauka Publ., 1973. 448 p.

13. Pontryagin L. S., Boltyansky V. G., Gamkrelidze R. V., Mishchenko E. F. Matematicheskaya teoriya optimal'nykh protsessov [Mathematical Theory of Optimal Processes]. Moscow, Nauka Publ., 1983. 393 p.

14. Dykhta V. A., Antipina N. V., Samsonyuk O. N. Optimal'noe upravlenie v ekonomike: prosteishie modeli [Optimal control in economy: simplest models]. Irkutsk State University Publ., 1998. $115 \mathrm{p}$.

15. Egorov A. I. Obyknovennye differentsial'nye uravneniya s prilozheniyami [Ordinary differential equations with applications]. $2^{\text {nd }}$ ed. Moscow, Fizmatlit Publ., 2005. 384 p.

\section{Информация об авторе}

Антипина Наталья Валерьевна - кандидат физико-математических наук, доцент, кафедра математики и эконометрики, Байкальский государственный университет, 664003, г. Иркутск, ул. Ленина, 11, e-mail: natant2012@mail.ru.

\section{Author}

Natalya V. Antipina - PhD in Physics and Mathematics, Associate Professor, Chair of Mathematics and Econometrics, Baikal State University, 11 Lenin St., 664003, Irkutsk, Russian Federation; e-mail: natant2012@mail.ru.

\section{Библиографическое описание статьи}

Антипина Н. В. Влияние инвестиционной составляющей на экономические показатели малых и средних фирм / Н. В. Антипина // Baikal Research Journal. — 2017. — T. 8, № 2. - DOI: 10.17150/2411-6262.2017.8(2).26.

\section{Reference to article}

Antipina N. V. Influence of investment constituent on economic indicators of small and medium-sized firms. Baikal Research Journal, 2017, vol. 8, no. 2. DOI: 10.17150/24116262.2017.8(2).26. (In Russian).

\section{Baikal Research Journal}

